Portland State University

PDXScholar

\title{
Improved Complexity of Quantum Oracles for Ternary Grover Algorithm for Graph Coloring
}

\author{
Marek Perkowski \\ Portland State University, marek.perkowski@pdx.edu \\ Yushi Wang
}

Follow this and additional works at: https://pdxscholar.library.pdx.edu/ece_fac

Part of the Electrical and Computer Engineering Commons Let us know how access to this document benefits you.

\section{Citation Details}

Perkowski, Marek and Wang, Yushi, "Improved Complexity of Quantum Oracles for Ternary Grover Algorithm for Graph Coloring" (2011). Electrical and Computer Engineering Faculty Publications and Presentations. 204.

https://pdxscholar.library.pdx.edu/ece_fac/204

This Conference Proceeding is brought to you for free and open access. It has been accepted for inclusion in Electrical and Computer Engineering Faculty Publications and Presentations by an authorized administrator of PDXScholar. Please contact us if we can make this document more accessible: pdxscholar@pdx.edu. 


\section{Improved Complexity of}

Quantum Oracles for

\section{Ternary Grover}

Algorithm

\section{for Graph Coloring}

Yushi Wang

Marek Perkowski

mperkows@cecs.pdx.edu 


\section{Goals of our research}

- Mathematicians who create quantum algorithms (Grover, Shor, Phase Estimation, Many Body Dynamics, etc) do not pay attention to how the circuit is designed and to its complexity

- They write: "let be given Unitary matrix U"

- We want to create practical (gate level) quantum algorithms for practical problems, such as graph coloring or SAT 


\section{Goals of our research}

- Compare binary versus MVL quantum algorithms

1) Yale Fan - 2007 Oslo

2) Yushi Wang - 2011 Tuusula

3) Vamsi Parasa - 2011 Tuusula

- Compare one-hot and minimum length binary encodings

1) Sidharth Dhawan - 2011 Tuusula

- Compare various multi-valued encodings.

- Compare various non-minimum length binary encodings.

- Compare various non-minimum length MVL encodings.

- Do these comparisons on realistic quantum costs, on several practical examples 


\section{Map and Graph Coloring Problem}

- Map coloring problem:

- Given a map with disjoint regions, called countries, color the countries

- No two countries may be of the same color

- Minimal number of colors desired.

- Graph coloring

- In a map, replace all countries with nodes, and

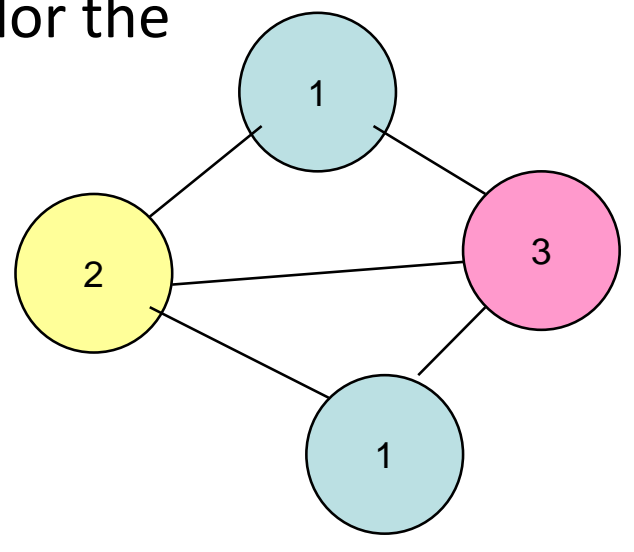
boundaries with lines

- Resulting planar graph represents the map.

- In general, graph is not planar, which means that the chromatic number can be as high as the number of nodes. 


\section{Graph coloring problem}

- Represent colors as strings (binary or otherwise)

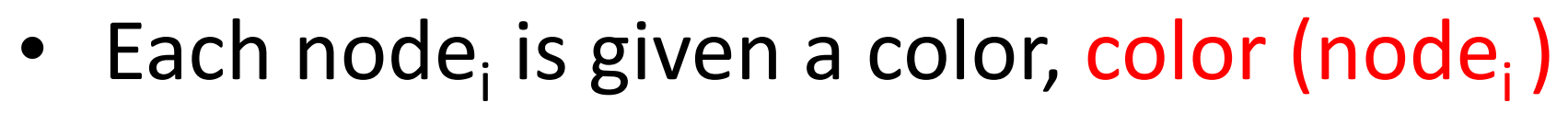

- Adjacent nodes are compared.

- If color $\left(\right.$ node $\left._{a}\right)=$ color $\left(\right.$ node $\left._{b}\right)$, bad coloring

- If color $\left(\right.$ node $_{\mathrm{a}}$ ) $\neq$ color $\left(\right.$ node $_{\mathrm{b}}$ ), for all adjacent nodes, good coloring 
- The Graph Coloring Grover Algorithm has several applications in logic synthesis, scheduling, allocation, planning, robot motion, robot communication, resource allocation, conflict resolution, floor-planning, and many others.

- It can serve as a "generic CSP solver" similar to a general SAT solver. 


\section{Quantum Unstructured Search ("Grover")}

- Grover's Algorithm uses superposition property of quantum information to perform an unstructured search more quickly

- The initial input qubits are superposed to represent all possible solutions

- The Oracle operation tags the phase of the solution states in this superposition

- Another circuit then changes the phase information (which is hidden) into amplitude information (which we can detect). inversionabout-the-average operation $\mathrm{HZH}$

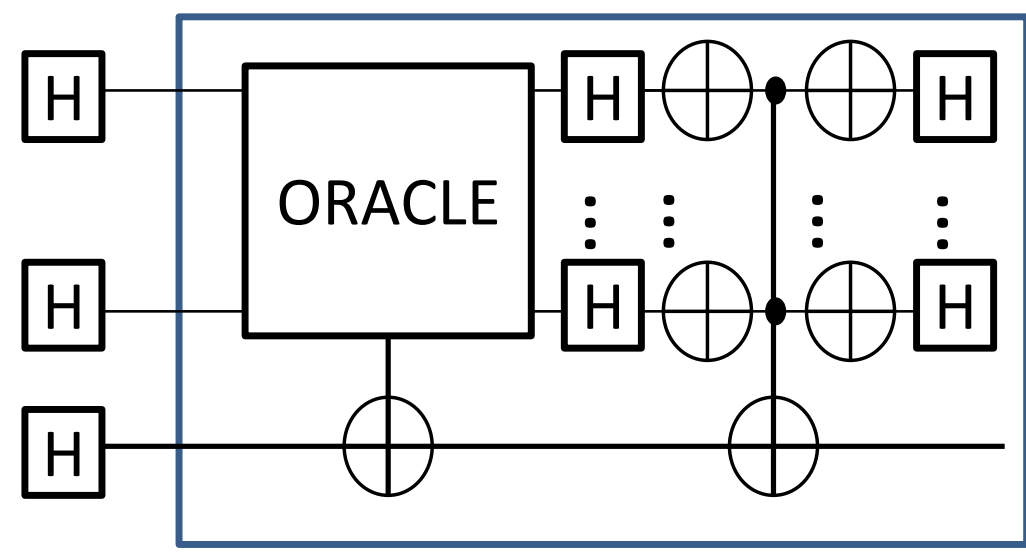

Grover Loop (* VN)

Superposed amplitudes of all states

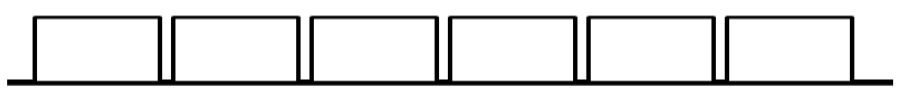

- Uses permutative Grover's Oracle based on comparators for edges and a global AND gate.

- This process is iterated VN times (as opposed to $\mathrm{N}$ iterations in classical logic) to maximally amplify the states.

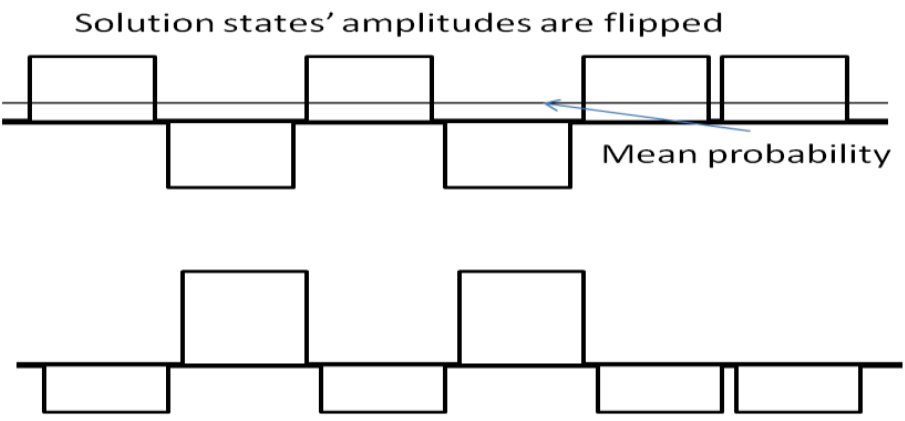

Solution states are amplified, while nonsolutions are less probable 


\section{Circuit level representation of Grover}

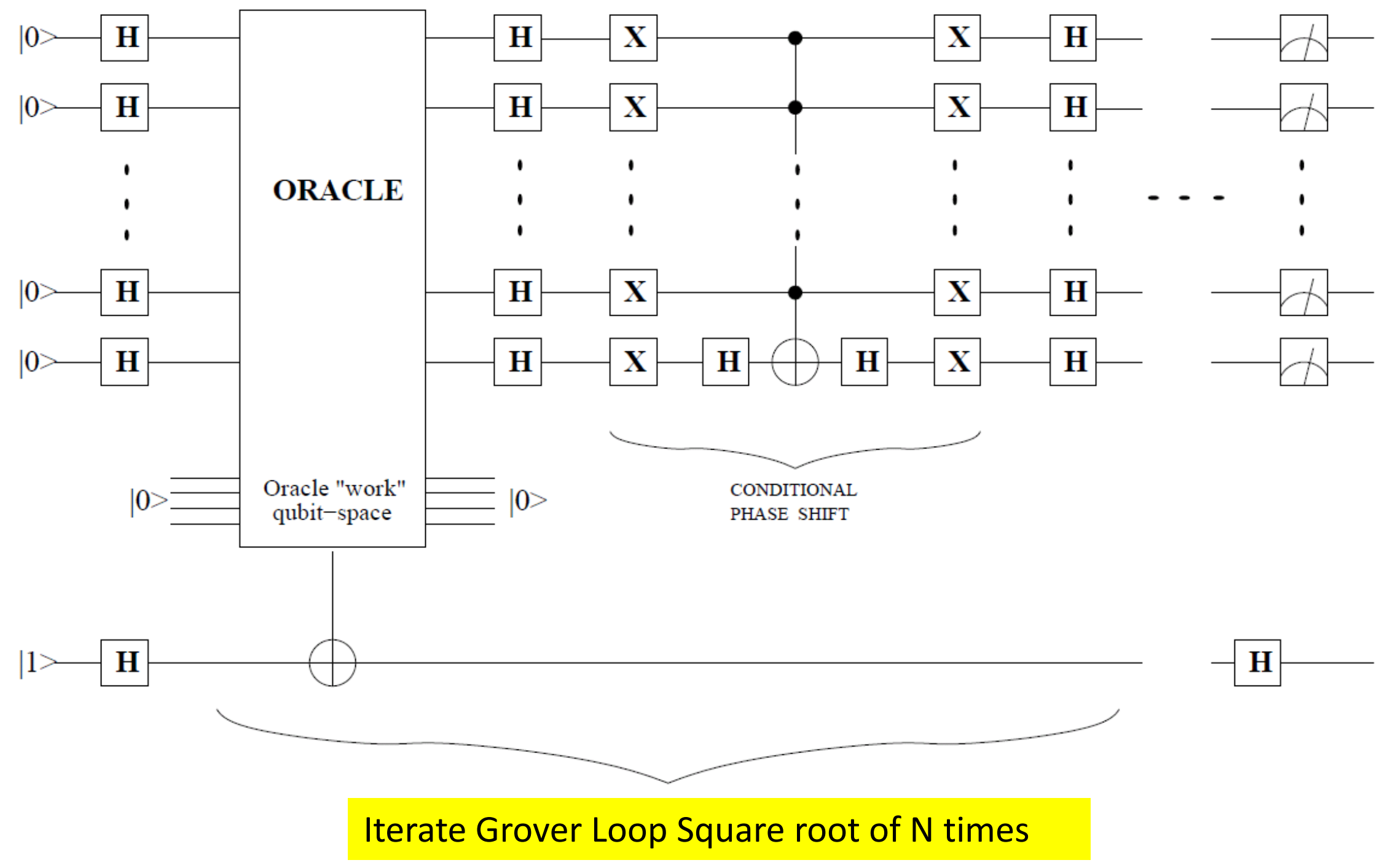




\section{Concept of the coloring oracle}

- Since there are three nodes, each can take on up to three colors

- Each node is represented with || $\log _{2}(3)||=2$ qubits so that the total number of possible collective states per node is

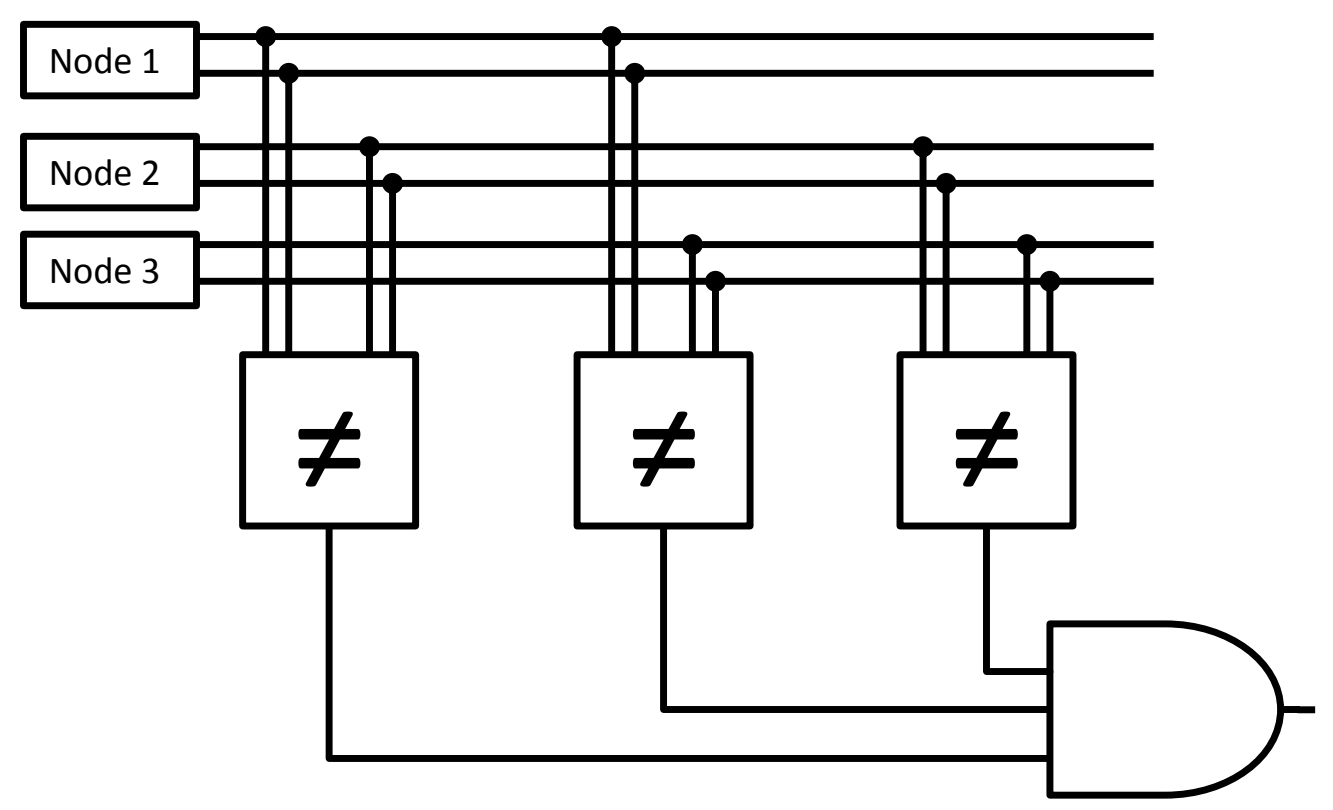
more than three.

- For example, the states $\mid 00>$ and $\mid 01>$ represent different colors.

- I have to make sure no two nodes that are connected are assigned the same color using bit-by-bit inequality gates. 


\section{Ternary Quantum Logic}

- Qutrits instead of qubits; superpositions are now of states

$|0\rangle,|1\rangle$, and $|2\rangle$

- Chrestenson gate is used in place of Hadamard gate:

$C H=\frac{1}{\sqrt{3}}\left[\begin{array}{ccc}1 & 1 & 1 \\ 1 & d 1 & d 2 \\ 1 & d 2 & d 1\end{array}\right]$, where $d 1=-(1+d 2)=-\frac{1}{2}(1-\sqrt{3} i)=e^{\frac{2 \pi i}{3}}$

- Controlled-NOT binary gates replaced by single-qutrit reversible functions:

Controlled(+1), controlled(+2), controled(01),

Controlled(02), and controlled(12)

ISMVL 2011, 23-25 May 2011, Tuusula, Finland 


\section{9 colors, \\ 4 qubits \\ 9 colors, \\ 2 qutrits}

0000

0001

0010

0011

0100

0101

0110

0111

1000
00

01

02

10

11

12

20

21

22
Worst case

9 nodes $=9 * 8 / 2=36$ edges

$36 * 4$-qubit comparators

PLUS final Toffoli gate with four inputs in AND

\section{Versus}

36 2-qutrit comparators

PLUS final Toffoli gate with

2 inputs in AND

PLUS global Toffoli with 36 INPUTS, this gate can be internally built as binary or ternary, but realizes binary logic AND 


\section{Ternary Grover's}

- In 2007, Yale Fan proposed a design for a generalized multivalued Grover's Algorithm

- For base $n$, a vector of quantum Fourier transforms (base $n$ ) is applied to the vector of $|0\rangle$ states. (Chrestenson Transform).

- The HZH has been replaced by diffusion operator

$$
D=\left(1-\omega^{-1}\right)|\psi\rangle\langle\psi|-I
$$

$\mathrm{CHZCH}$

ISMVL 2011, 23-25 May 2011, Tuusula, Finland 


\section{Oracle overview: a general case}

- Evaluates a coloring of a map by comparing each pair of connected nodes.

- Each comparison gives either 1 (good coloring of edge) or 0 (bad coloring)

- This is inequality comparator. Output is 1 when colors are different.

- If all of these comparisons are good, overall output is 1 . If the two neighboring nodes have he same color, output $=0$.

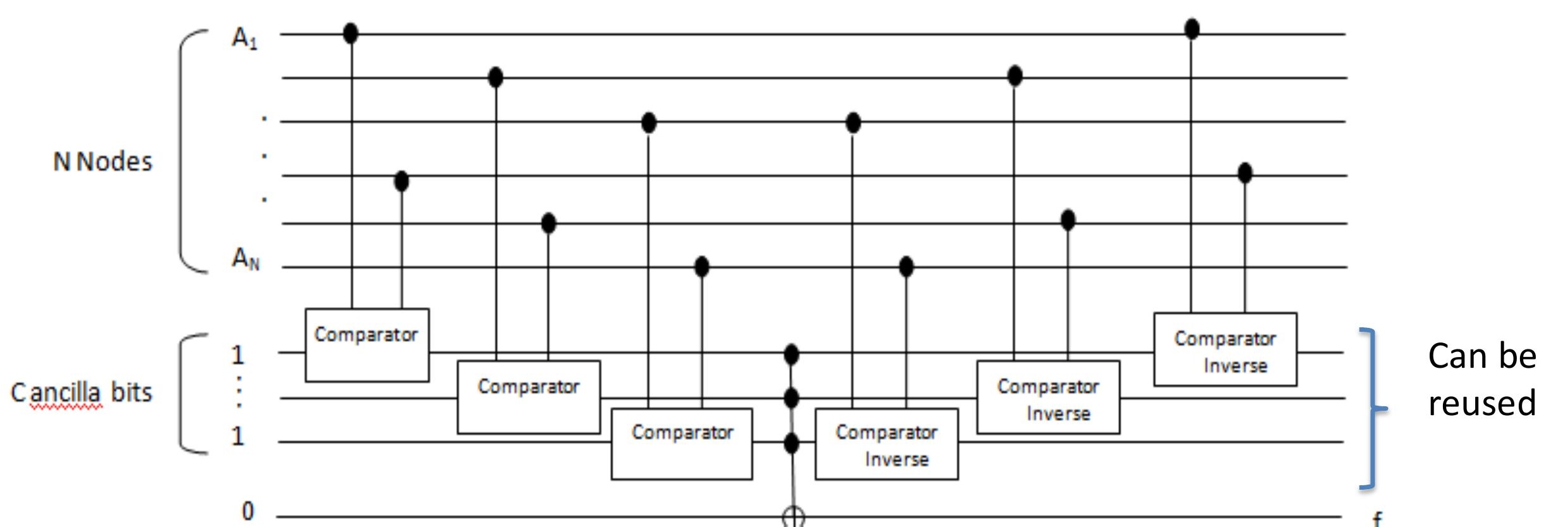




\section{Binary Inequality Comparator circuit}

- Compare two strings of digits and operates on an ancilla bit.

- If the two strings are exactly the same, output is flipped from 1 to 0.

- If the two inputs are not exactly the same, output $=1$.

- After comparison, the operations are mirrored to return ancilla bit to 1

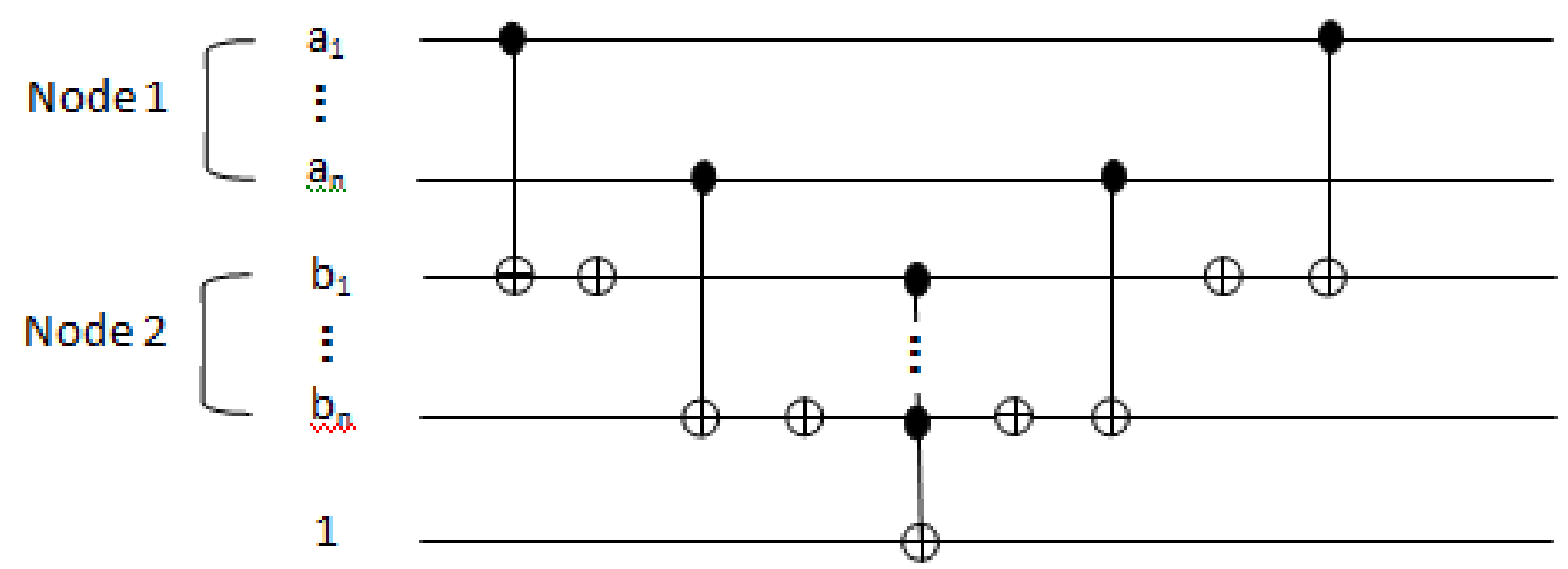




\section{Costs of binary vs ternary quantum comparators}

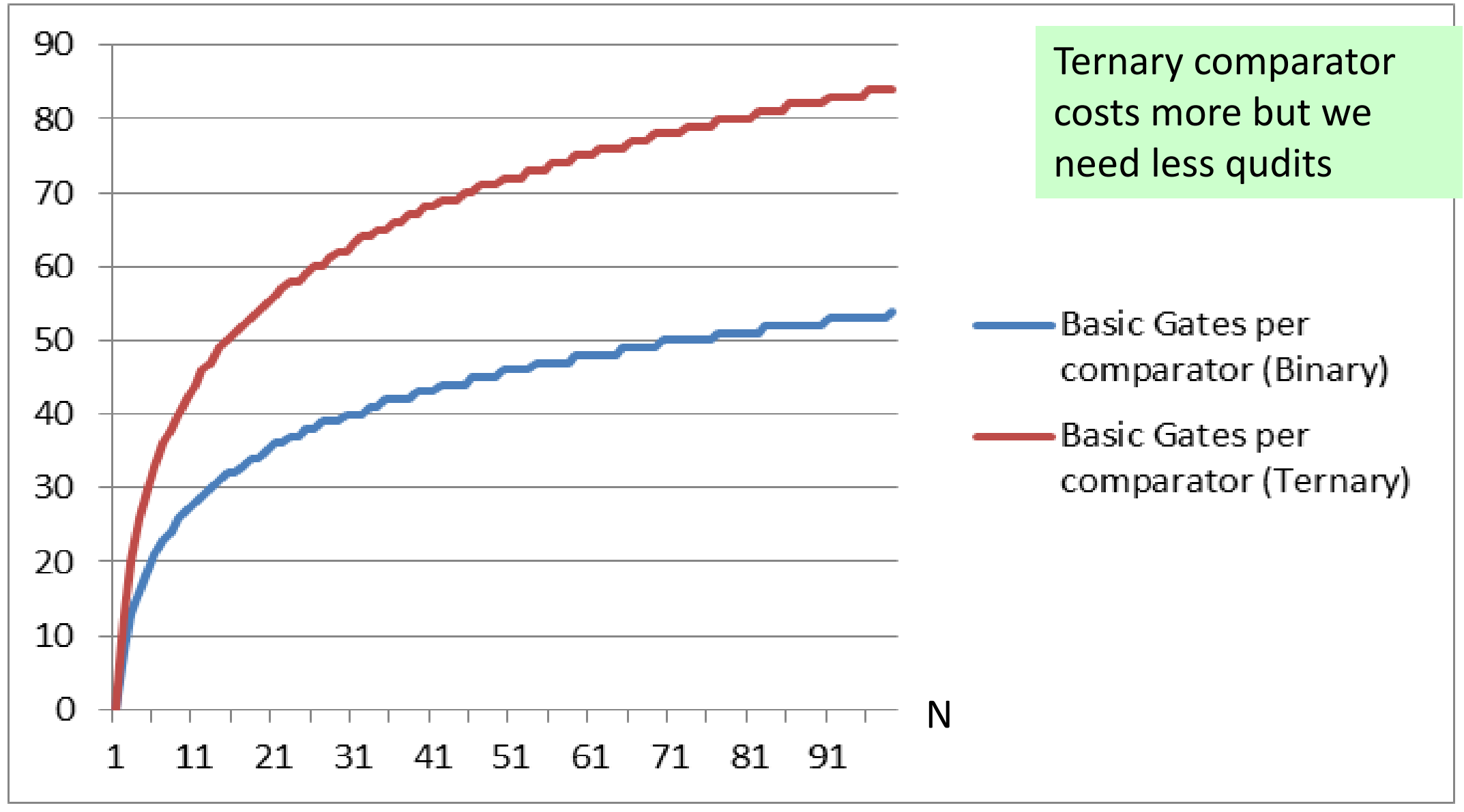

Figure 7 - Graphic Description of the cost of Comparators, excluding Toffoli.

ISMVL 2011, 23-25 May 2011, Tuusula, Finland 


\section{Costs of binary Toffoli gate with many inputs}

- In [3], the cost of the binary $n$-bit Toffoli gate was found to be one of the following:

- For a small number of input gates, such as from 2-5 inputs, the optimal construction of the Toffoli gate currently costs $2^{n}-3$ basic quantum operations.

- For larger numbers, the cost grows linearly, at 32n-96 gates, plus one garbage bit per input.

- Thus, the complexity of the Toffoli gate overall grows linearly. 


\section{NEW -- Ternary Toffoli}

- As shown in previous diagram, a ternary version of the Toffoli gate (multi-controlled NOT gate)

- Proposed multi-controlled NOT gate operates on one ancilla bit and any number of inputs, like the binary Toffoli

- If all inputs equal 1 , output is 1 . Otherwise, output $=0$

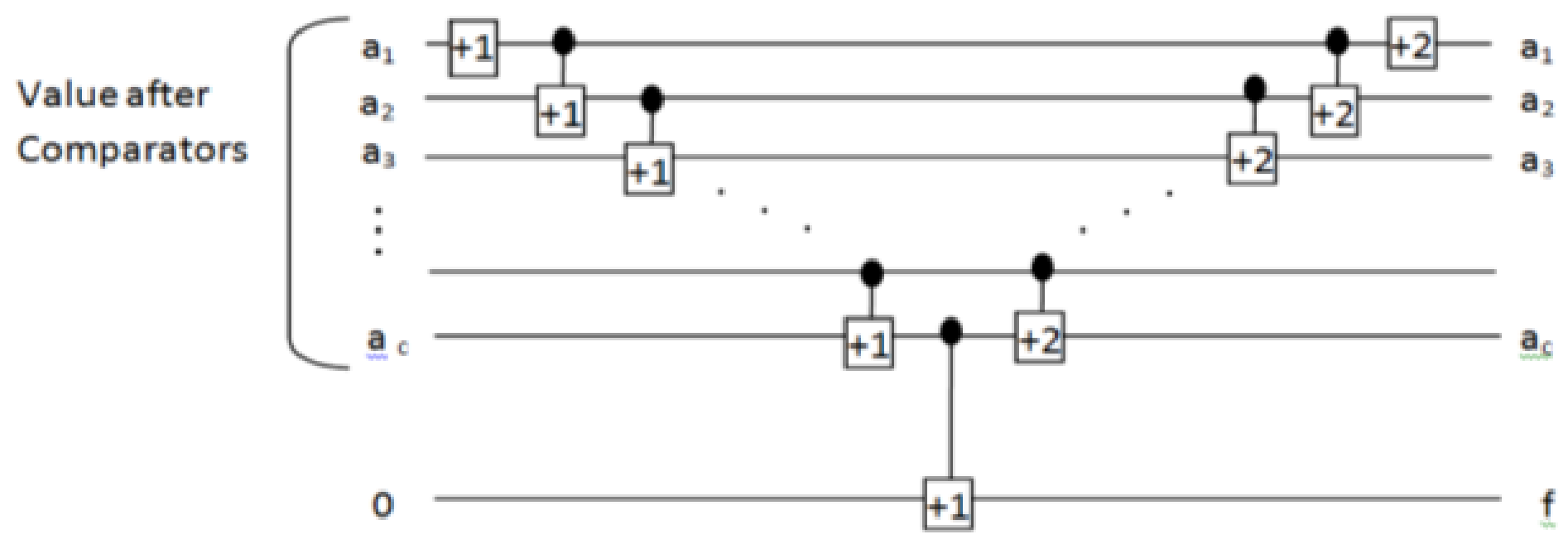




\section{The reduced cost of the new Toffoli gate}

- Using the same standard cost of 2-qudit gates, the calculated cost of the multi-valued Toffoli gate is significantly lower.

- Using the above diagram, one can construct a multi-valued extension using only $2 n+1$ gates, which is also linear, but at $1 / 8$ of the binary version's cost.

- A graphic comparison of the three is shown in next figure. 


\section{Costs of Toffoli gates}

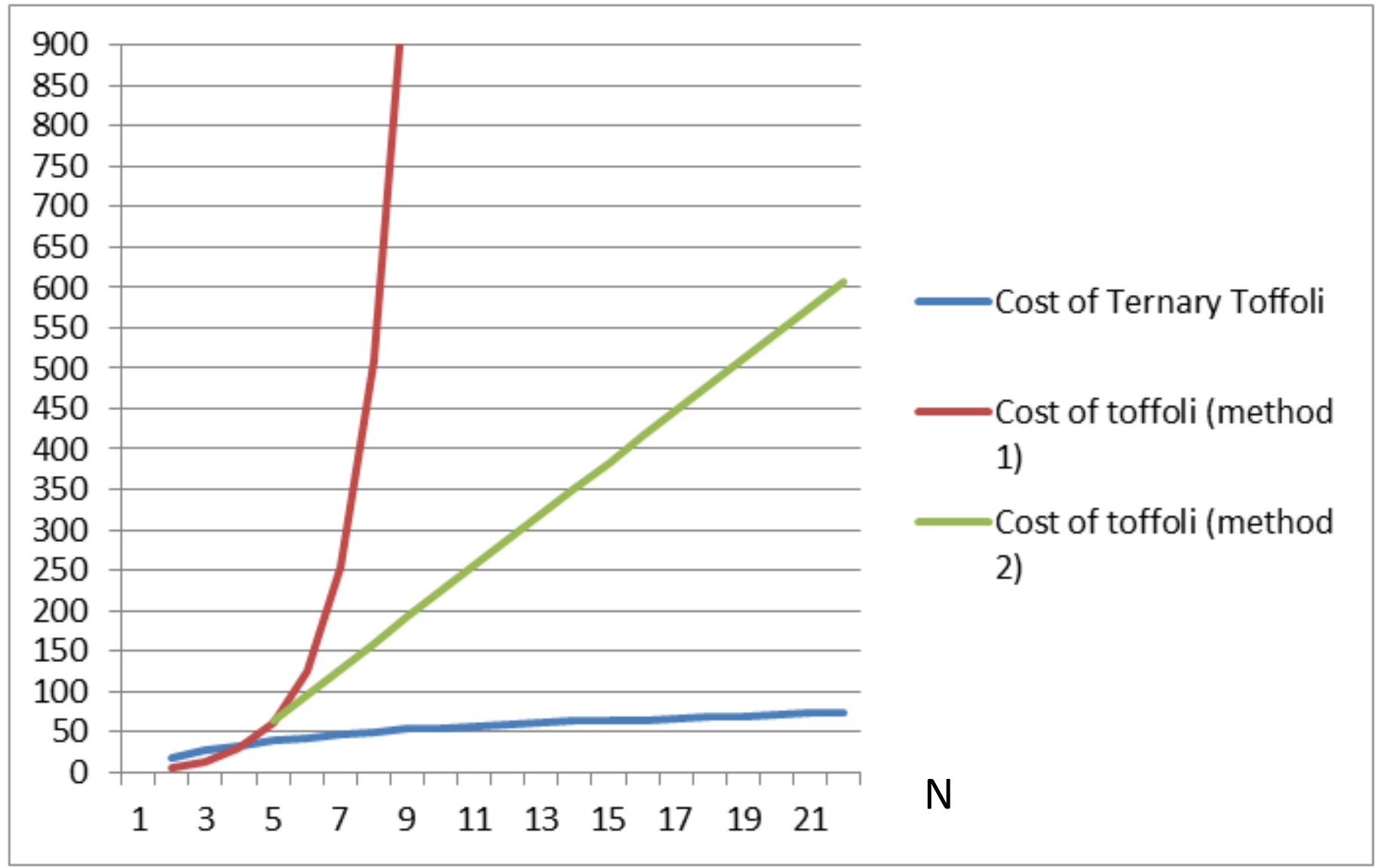

Figure 6-Graphic Description of the cost of Toffoli gates

ISMVL 2011, 23-25 May 2011, Tuusula, Finland 
Final cost comparison of binary and

\section{ternary comparators}

- Using the formulae for the cost of Toffoli in terms of two-qubit gates, the cost jumps significantly to $4\left(\log _{2} N\right)+32\left(\log _{2} N\right)-96$ gates, assuming $N \geq 5$.

- For $\mathrm{N}<5$, the cost is $4\left(\log _{2} N\right)+2^{(\log 2 N)}-3$ gates.

- Now factoring the cost of Toffoli gates into the equation, the cost becomes much more favorable towards ternary logic, as shown in next Figure. 


\section{The final total costs: binary vs ternary}

- Using the diagrams of the mirrored comparators, we can see that the cost of the ternary comparator is

$>34\left(\log _{3} N\right)$ two-qutrit gates,

$>4\left(\log _{3} N\right)$ single-qutrit gates,

$>$ and one Toffoli gate with $\log _{3} N$ inputs.

- The binary comparator costs $4\left(\log _{2} N\right)$ two-qubit gates plus one $\log _{2} \mathrm{~N}$-input Toffoli gate. 


\section{Costs of comparators. With Toffoli}

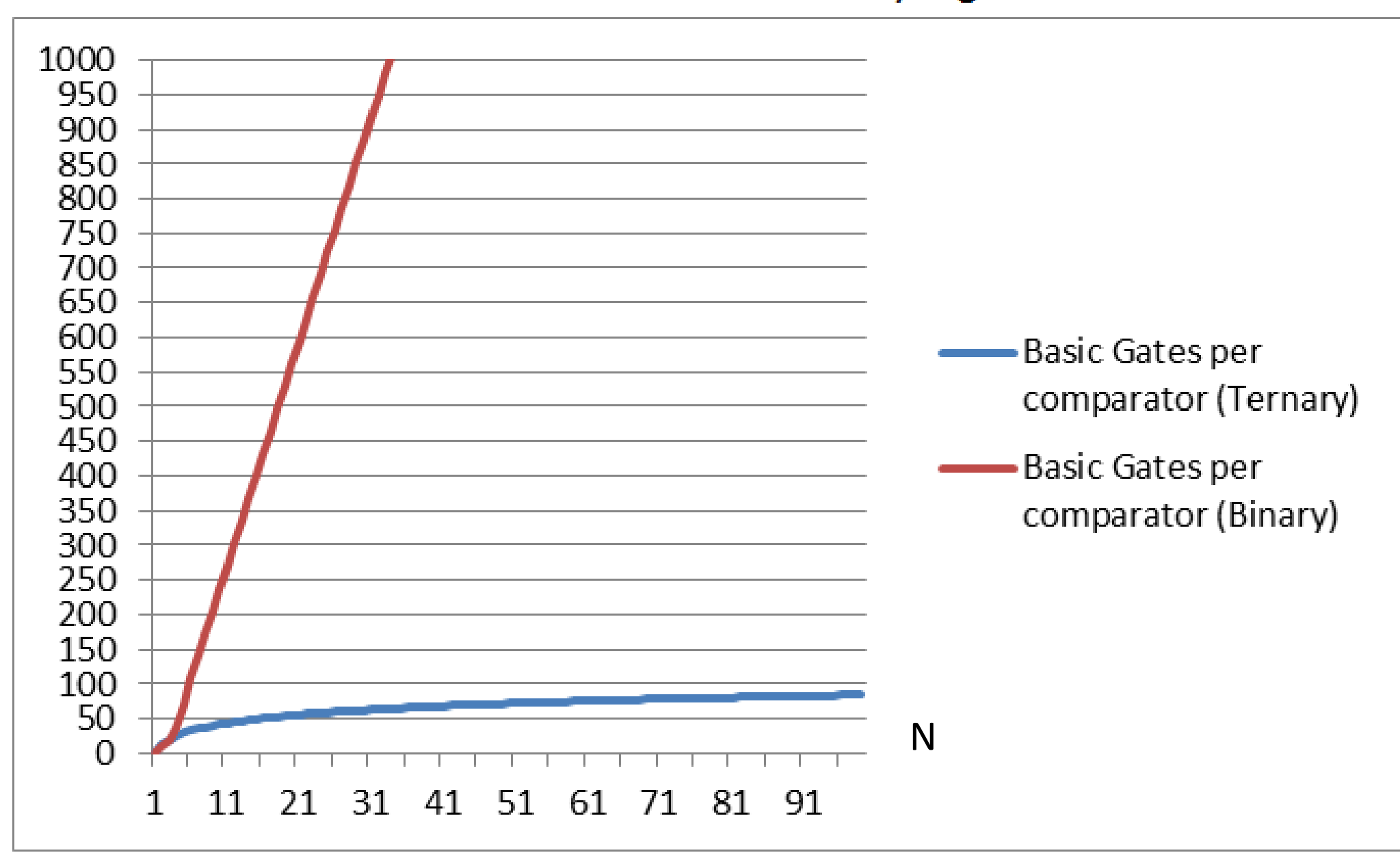

Figure 8 - Graphic Description of the cost of Comparators, including Toffoli.

ISMVL 2011, 23-25 May 2011, Tuusula, Finland 


\section{Simulation}

- Simulations have been carried out in MATLAB

- Since MATLAB isn't particularly good with large matrices, simulations have been limited to small cases.

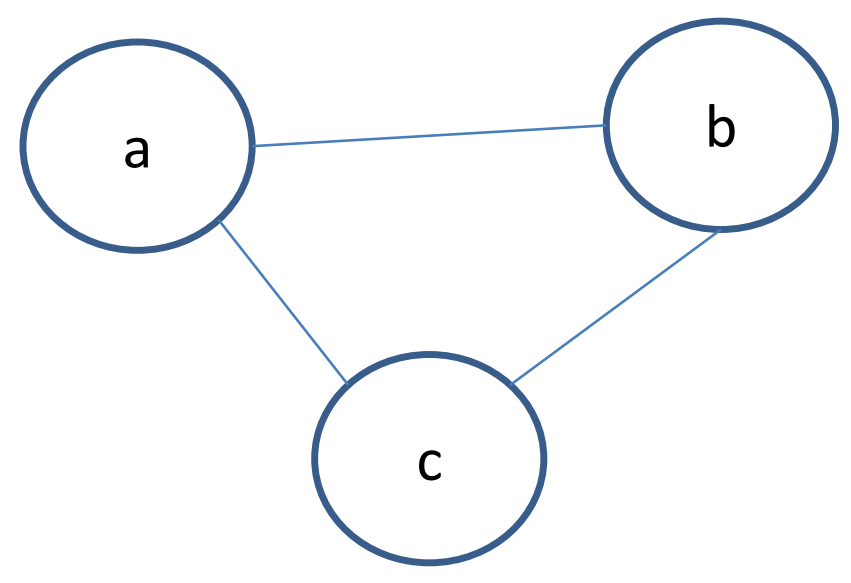

ISMVL 2011, 23-25 May 2011, Tuusula, Finland 


\section{Simulation (cont'd)}

- Overall layout of the simulated circuit

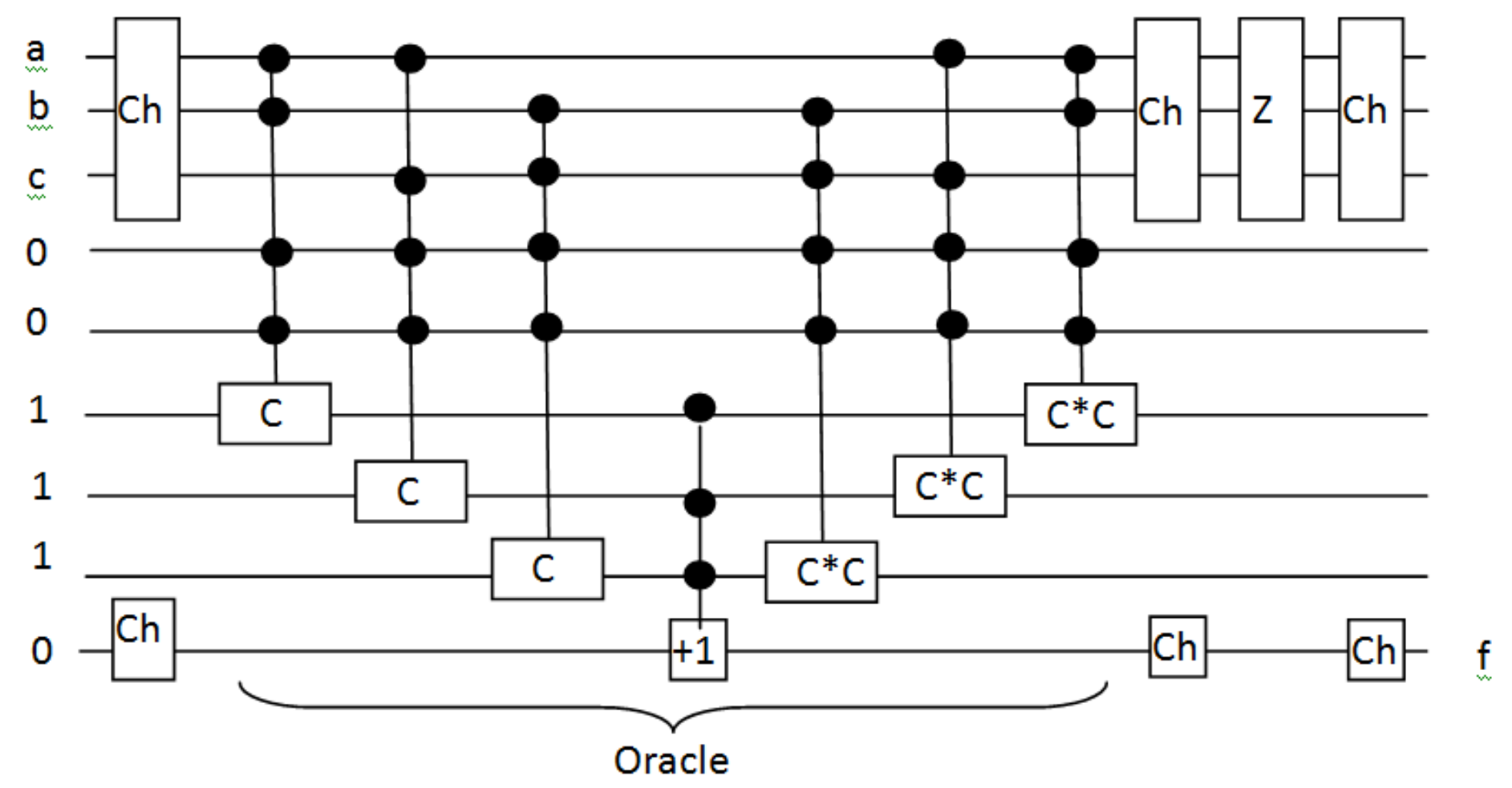

ISMVL 2011, 23-25 May 2011, Tuusula, Finland 


\section{Simulation (cont'd)}

- MATLAB requires no wire crossovers, so the simulation used a slightly modified version of the ternary comparator circuit

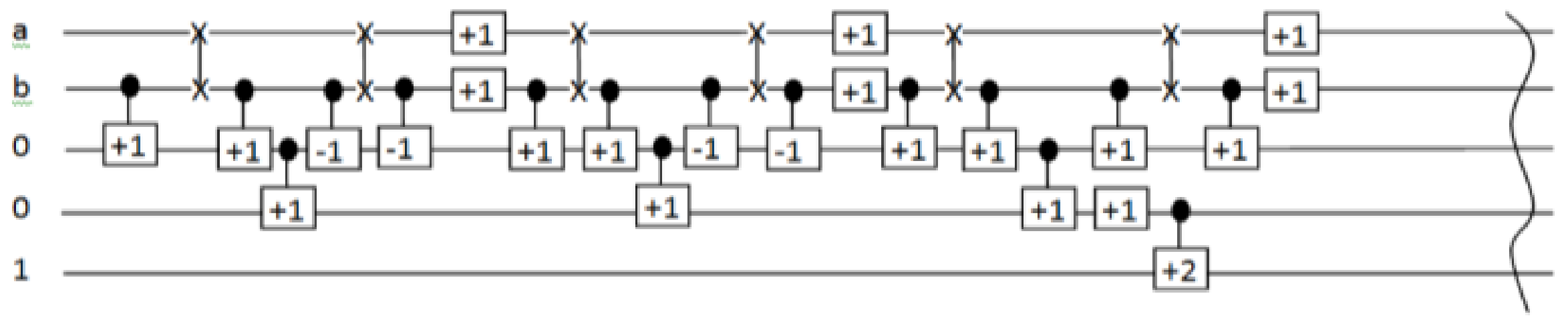

Linear nearest neighbor model 


\section{Grover Iterations}

- Grover Algorithm has been shown to require approximately $V M$. iterations rounded up, where $M$ is the number of "items in database".

- Since we have 3 nodes, each with 3 states, the total number of items is $3^{3}$, or 27.

- Thus, our solution algorithm should have $\sqrt{ } 27=6$ iterations of the Grover operator.

- With each successive application of the operator, the probabilities of the "good" states should increase in amplitude, whereas the "bad" states should decrease in amplitude until they reach $\sim 0$. 


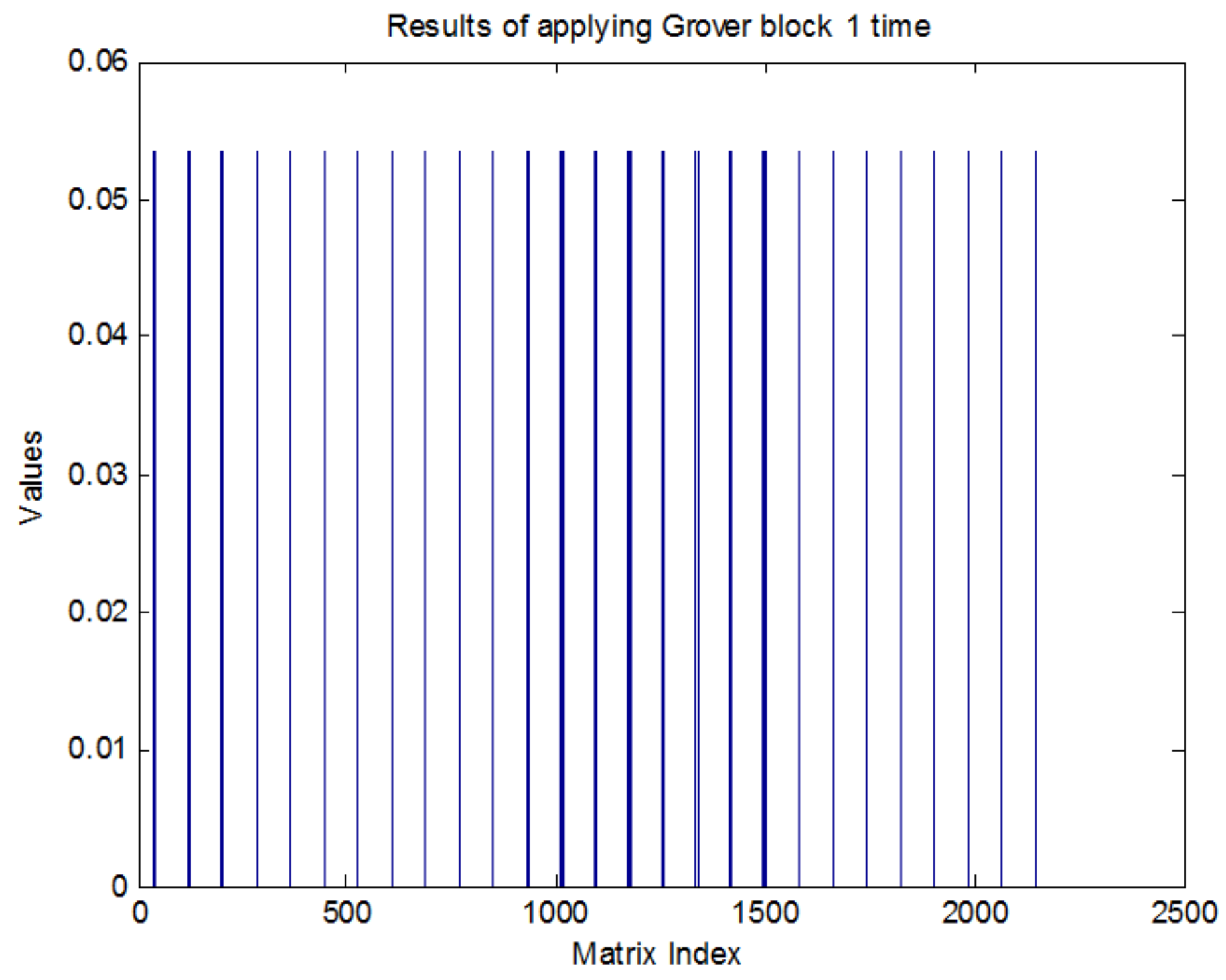

ISMVL 2011, 23-25 May 2011, Tuusula, Finland 


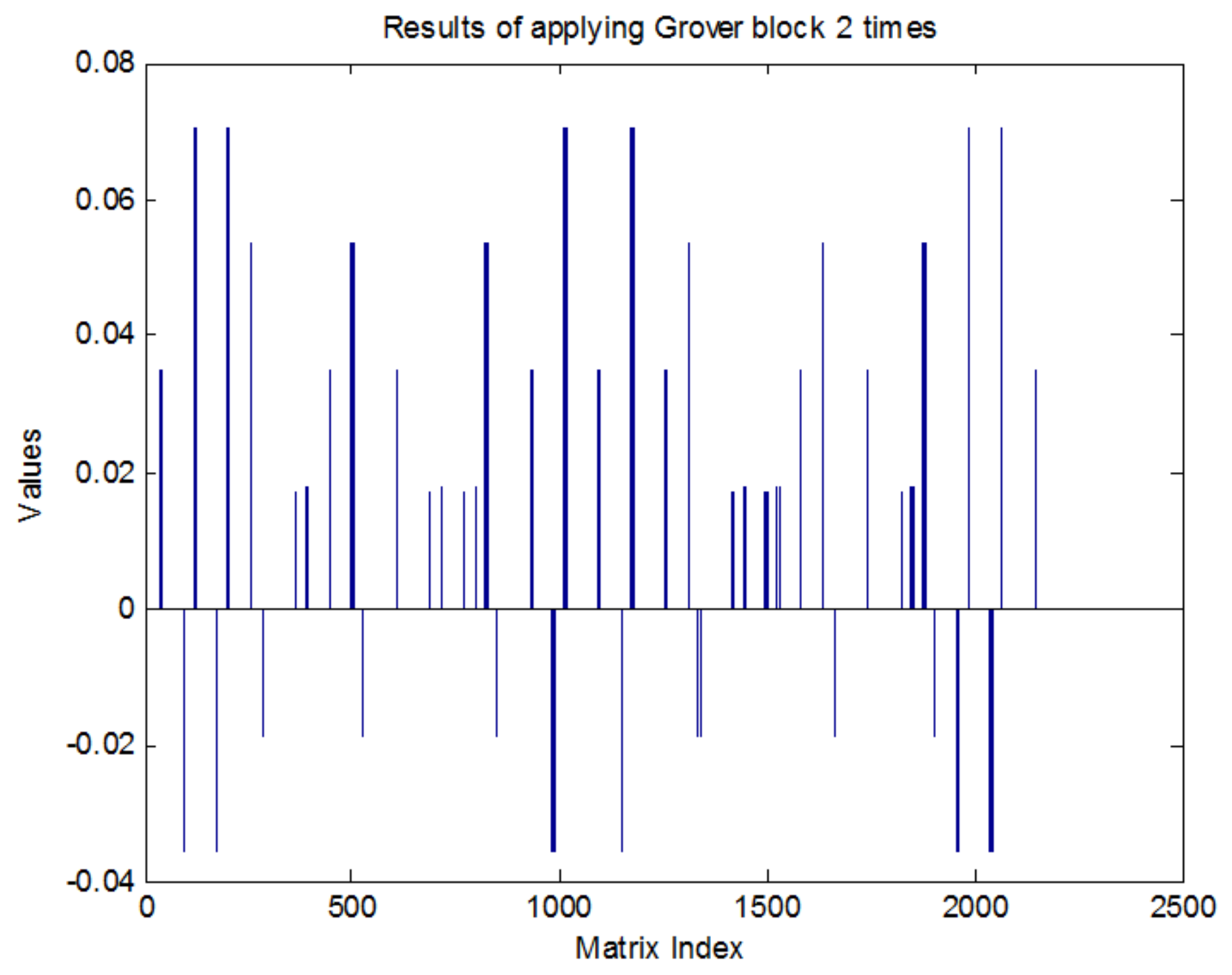

ISMVL 2011, 23-25 May 2011, Tuusula, Finland 


\section{After 6 iterations}

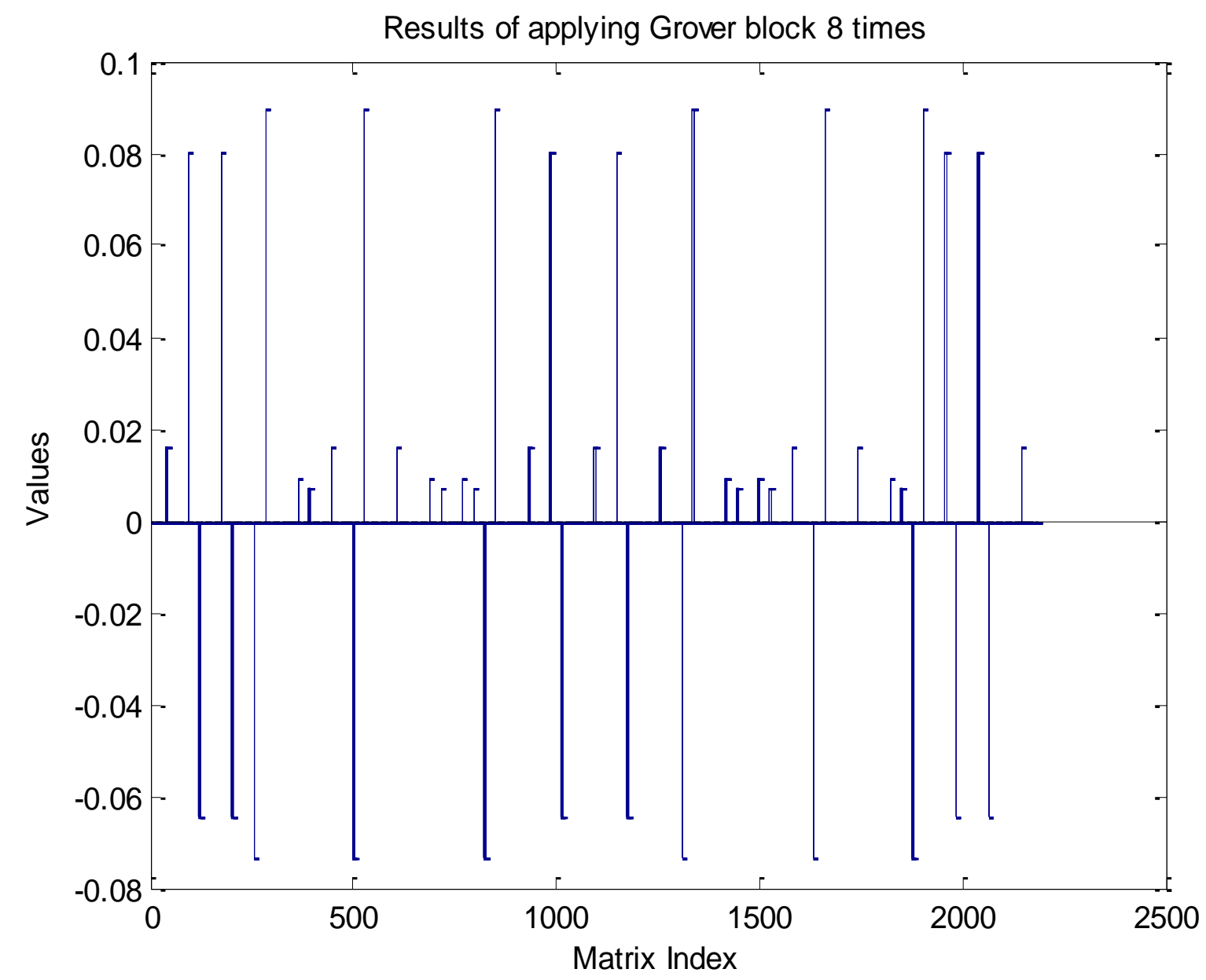




\section{How many iterations?}

- After 2 Grover loops, the highest amplitude of the output was .655,

- whereas after 6 Grover loops, the highest amplitude had increased to .87 . 


\section{Conclusions 1}

- We synthesized a binary quantum algorithm for a graph coloring problem from binary gates

- We synthesized a ternary quantum algorithm to a graph coloring problem from ternary gates

- The ternary oracle can solve NP-complete problems, the same as binary

- We compared the complexities of binary and ternary oracles for solving arbitrary size graph-coloring problems.

- The ternary version of the oracle was shown to be able to perform the same functions as the binary functions equally well, giving the same outputs. (simulation)

- The ternary oracle has a lower cost in wires and garbage bits because of its ability to store more information per qudit

- The ternary oracle has smaller quantum cost.

ISMVL 2011, 23-25 May 2011, Tuusula, Finland 


\section{Conclusions 2}

- The number of gates required to build the ternary oracle is initially higher than that of the binary oracle for small graphs.

- However, as the size and complexity of the graph grows, oracles in ternary-based logic have greater efficiency relative to binary oracles.

- The multi-valued extensions of binary oracles can be created for comparators, arithmetic circuits and Toffoli gates.

- Thus, any problem, even those requiring strictly binary outputs, can be solved with MV circuits.

- The circuits that are binary input / binary output can be built more efficiently with ternary logic inside.

ISMVL 2011, 23-25 May 2011, Tuusula, Finland 
Invention of a new binary Toffoli gate, ternary inside

- The ternary Toffoli gate, invented in this paper, performs a similar function and will be used as a building block for ternary logic synthesis in the future.

- Our new ternary Toffoli gate has $1 / 8$ the cost of the binary Toffoli gate, as it scaled linearly with the number of inputs with slope two, whereas the binary gate required 16 more 2-qubit gates for each additional input.

Value after Comparators

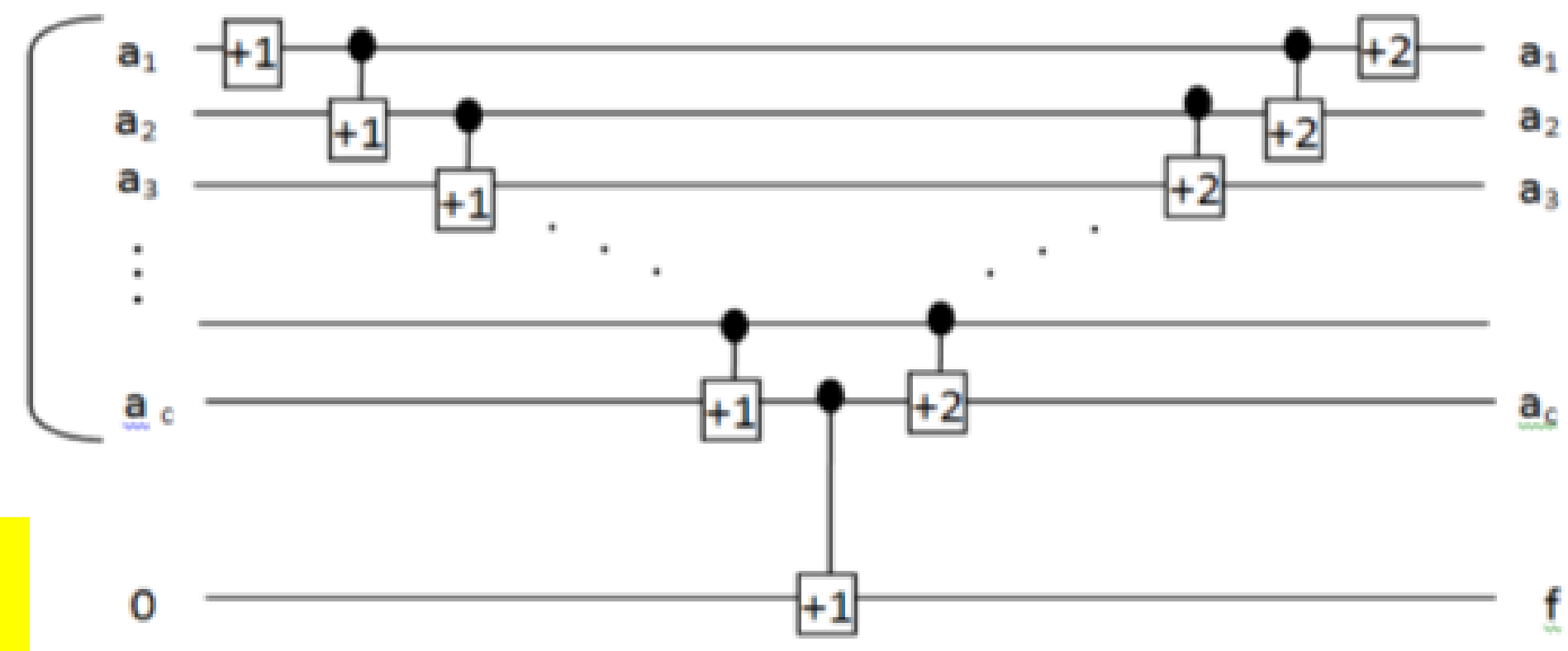




\section{Conclusions 4}

- Ternary gates cause different rotations than binary gates on particles.

- Therefore, the cost in NMR (Nuclear Magnetic Resonance) pulses of 2-qudit gates in binary and ternary are not the same.

- Even 2-qubit binary gates require different numbers of pulses from each other, such as the Feynman gate, which requires 5 pulses, compared to the $V$ and $V^{+}$gates, which require 3 pulses each.

- Ternary gates, since they operate with $120^{\circ}$ rotations, would have still different numbers of pulses, which may affect their efficiency relative to binary gates.

ISMVL 2011, 23-25 May 2011, Tuusula, Finland 


\section{Conclusions 5}

- For some future Constrained Satisfaction Problems for Grover, the oracle should have mixed (hybrid) qudits for reduction of quantum cost and processing speed.

- Synthesis methods for hybrid quantum circuits and binary external/multiple-valued internal quantum circuits should be created

- Ternary results will be even better for the Nearest Linear Nearest Neighbor Model, as the ternary comparators are more compact and ternary solution requires less SWAP gates to be added.

On the other hand, the ternary SWAP gates are more expensive in terms of pulses.

ISMVL 2011, 23-25 May 2011, Tuusula, Finland 